\title{
Synchronization of Complex Dynamical Networks with Nonidentical Nodes and Derivative Coupling via Distributed Adaptive Control
}

\author{
Miao Shi, Junmin Li, and Chao He \\ Department of Mathematics, Xidian University, Xian 710071, China \\ Correspondence should be addressed to Miao Shi; miaomiao_90@aliyun.com
}

Received 23 May 2013; Revised 19 August 2013; Accepted 19 August 2013

Academic Editor: Jun $\mathrm{Hu}$

Copyright (C) 2013 Miao Shi et al. This is an open access article distributed under the Creative Commons Attribution License, which permits unrestricted use, distribution, and reproduction in any medium, provided the original work is properly cited.

\begin{abstract}
Adaptive synchronization control is proposed for a new complex dynamical network model with nonidentical nodes and nonderivative and derivative couplings. The distributed adaptive learning laws of periodically time-varying and constant parameters and distributed adaptive control are designed. The new method which can obtain the synchronization error of closed-loop complex network system is asymptotic convergence in the sense of square error norm. What is more, the coupling matrix is not assumed to be symmetric or irreducible. Finally, a simulation example shows the feasibility and effectiveness of the approach.
\end{abstract}

\section{Introduction}

Complex dynamical networks have become a focus issue and have attracted increasing attention in many fields. The structure of many real systems in nature can be modeled by complex networks, such as biological neural networks, ecosystems, food webs, and World Wide Web $[1,2]$. It has been demonstrated that many cooperative behavior mechanisms and complex phenomena in nature and society have close relationships with network synchronization [3]. Thus, the synchronization of complex systems has been widely studied, and many control schemes, such as adaptive control $[4,5]$, sliding mode control [6], and state observer-based control [7], have been focused on this topic. However, many existing works on synchronization are based on the networks with same nodes. Since almost all complex dynamical networks in engineering have different nodes, it is unrealistic to assume that all network nodes are the same. For example, in a multirobot system, the robots can have distinct structures or have different parameters such as masses and inertias. With much more complicated behaviors, the synchronization schemes for complex networks with identical nodes cannot be directly applied to the dynamical networks with nonidentical nodes. Therefore, it is strongly necessary to conduct further investigation of new synchronization schemes for a complex dynamical network with nonidentical nodes.

Recently, some papers have studied the synchronization problem with nonidentical nodes. A pinning control scheme was developed to realize the cluster synchronization of community networks with nonidentical nodes [8]. Moreover, other weaker forms of synchronization, namely, output synchronization and bounded synchronization, were proposed in $[9,10]$. It is so difficult for a complex network with nonidentical nodes to achieve asymptotic synchronization that little work has been reported to deal with the problem. By constructing a common Lyapunov function for all the nodes, a synchronization criterion for a nonidentical nodes system was given in [11]. Some local robust controllers were designed for the network with strictly different nodes to achieve generalized synchronization in [12]. In [13], the asymptotic synchronization for complex dynamical networks with nonidentical nodes was studied. Based on solving a number of lower dimensional matrix inequalities and scalar inequalities, global synchronization criteria were given. Except the propriety of nonidentical nodes, the time-varying parameter and unknown system parameters should be considered meanwhile. Reference [14] introduced the adaptive synchronization of complex network systems 
with unknown time-varying nonlinear coupling. Although it considered nonlinearly parameterized complex dynamical networks with unknown time-varying parameters, the nonidentical nodes were not considered yet.

On the other hand, the aforementioned papers only considered nonderivative coupling. Taking into account the complexity of the network, such as population ecology [15] and robots in contact with rigid environments [16], there always exist delayed coupling [17], derivative coupling, and so on. In [18], the authors proposed a general complex dynamical network with non-derivative and derivative coupling. Under the designed adaptive controllers, the network system can asymptotically synchronize to a given trajectory. The paper was limited to the inner coupling matrix of derivative being identity matrix. A complex dynamic network model with derivative coupling and nonidentical nodes was studied in [19]. By designing the adaptive strategy with time derivative terms, the authors have dealt with the known derivative coupling successfully. However, they did not consider nonlinear parameterized complex network with time-varying coupling strength and the unknown derivative coupling strength.

In recent years, the adaptive learning control has been proposed to deal with the unknown constant or time-varying parameters of the system in order to obtain control input $[20,21]$. Under the designed adaptive learning control, the convergence of tracking error can be proved by using the composite energy function. Meanwhile, the sliding mode control (SMC) strategy has been successfully applied to a class of systems with nonlinearities and uncertainties [2224]. The advantage of using SMC method is the concepts of sliding mode surface design and equivalent control. In this paper, under the adaptive control strategy and inequality technique, we solve the proposed problem successfully. For the nonlinearities function, further work can focus on SMC method.

Motivated by the above discussion, the synchronization problem via new distributed adaptive control is proposed for nonlinear parametric complex dynamical networks with derivative coupling and nonidentical nodes. The main difficulty is how to deal with the unknown derivative coupling. Using the adaptive control and skill of inequality zoom, we find a way to overcome this challenge. By designing the distributed adaptive laws, the effect of derivative coupling can be eliminated. Based on a Lyapunov-Krasovskii-like composite energy function (CEF) $[25,26]$, the adaptive asymptotical synchronization is obtained for nonlinearly parameterized complex dynamical networks with nonidentical nodes and derivative coupling. The theoretical analysis shows that the designed effective distributed adaptive strategy guarantees that the system asymptotically stable in the $L_{T}^{2}$ norm sense and all closed-loop signals are bounded. And it is worth mentioning that the coupling configuration matrix may be arbitrary matrix with appropriate dimensions [27].

The rest of the paper is organized as follows. The problem formulation and preliminaries are given in Section 2. In Section 3, adaptive synchronization scheme of the complex dynamical networks is presented. In Section 4, a simulation example is provided to illustrate the effectiveness of the proposed controller. Finally, conclusion is given in Section 5.

\section{Problem Statement and Preliminaries}

A complex dynamical network consisting of $N$ nonidentical nodes is described as

$$
\begin{aligned}
\dot{x}_{i}(t)= & f_{i}\left(t, x_{i}(t)\right) \\
& +\sum_{j=1}^{N} a_{i j} \Gamma g\left(x_{j}(t), \varphi_{i}(t)\right) \\
& +\sum_{j=1}^{N} \alpha_{i} b_{i j} \Gamma \dot{x}_{j}(t), \quad i=1,2, \ldots, N,
\end{aligned}
$$

where $x_{i}(t)=\left[x_{i 1}(t), x_{i 2}(t), \ldots, x_{i n}(t)\right]^{T} \in R^{n}$ is the state vector of the $i$ th node, $f_{i}: R^{+} \times R^{n} \rightarrow R^{n}$ is a vectorvalued continuous function, $g: R^{n} \times R \rightarrow R^{n}$ is an unknown continuous nonlinear vector-valued function, $\varphi_{i}(t)$ represents the unknown time-varying function, and $\alpha_{i}$ is an unknown constant parameter. The inner coupling matrix $\Gamma=\left(\gamma_{i j}\right) \in R^{n \times n}$ is nonnegative definite. $A=\left(a_{i j}\right)_{N \times N}$ and $B=\left(b_{i j}\right)_{N \times N}$ are the coupling configuration constant matrices, which are defined as follows: if there is a connection from node $i$ to node $j(i \neq j)$, then $a_{i j}>0, b_{i j}>0$; otherwise, $a_{i j}=b_{i j}=0$. And the coupling matrices that satisfy $\sum_{j=1}^{N} a_{i j}=$ $\sum_{j=1}^{N} b_{i j}=0$.

Remark 1. In this paper, we assume that the strength of matrix $\Gamma$ is unknown, which is more general than the inner coupling matrices assumed to be diagonal matrix in [18]. As the authors in papers [19] and [21] only consider the known coupling matrix strength, it should be pointed out that the unknown derivative coupling consists of more information than the dynamical model in $[19,21]$. The coupling configuration matrices $A$ and $B$ need not be symmetric or irreducible. Thus, the results can be suitable to more complex dynamical networks.

Let $s(t) \in R^{n}$ be a solution of the dynamics of the isolated node to which all $x_{i}(t)$ are expected to synchronize.

Define synchronization error vectors

$$
e_{i}(t)=x_{i}(t)-s(t), \quad i=1,2, \ldots, N .
$$

In order to obtain the synchronized dynamics of network (1), we add an adaptive control strategy to nodes in network (1). Then, the controlled network is given by

$$
\begin{aligned}
\dot{x}_{i}(t)= & f_{i}\left(t, x_{i}(t)\right) \\
& +\sum_{j=1}^{N} a_{i j} \Gamma g\left(x_{j}(t), \varphi_{i}(t)\right) \\
& +\sum_{j=1}^{N} \alpha_{i} b_{i j} \Gamma \dot{x}_{j}(t)+u_{i}(t), \quad i=1,2, \ldots, N,
\end{aligned}
$$

where $u_{i}(t)=\left[u_{i 1}(t), u_{i 2}(t), \ldots, u_{i n}(t)\right]^{T} \in R^{n}, i=1,2, \ldots, N$ are the adaptive controllers to be designed. 
Since the row sum of coupling matrix is zero, we have the following dynamical error equation:

$$
\begin{aligned}
\dot{e}_{i}(t)= & \dot{x}_{i}(t)-\dot{s}(t) \\
= & f_{i}\left(t, x_{i}(t)\right) \\
& +\sum_{j=1}^{N} a_{i j} \Gamma\left(g\left(x_{j}(t), \varphi_{i}(t)\right)-g\left(s(t), \varphi_{i}(t)\right)\right) \\
& +\sum_{j=1}^{N} \alpha_{i} b_{i j} \Gamma\left(\dot{x}_{i}(t)-\dot{s}(t)\right)-\dot{s}(t)+u_{i}(t) \\
= & f_{i}\left(t, x_{i}(t)\right) \\
& +\sum_{j=1}^{N} a_{i j} \Gamma\left(g\left(x_{j}(t), \varphi_{i}(t)\right)-g\left(s(t), \varphi_{i}(t)\right)\right) \\
& +\sum_{j=1}^{N} \alpha_{i} b_{i j} \Gamma \dot{e}_{j}(t)-\dot{s}(t)+u_{i}(t) .
\end{aligned}
$$

Our objective is to design adaptive control laws $u_{i}(t)$ such that the synchronization errors converge to zero in the norm sense.

In order to derive the main results, some assumptions and lemmas are introduced as follows.

Assumption 2. For the unknown continuous function $g(\cdot, \cdot)$, the following inequality holds:

$$
\begin{aligned}
& \left\|g\left(x_{j}(t), \varphi_{i}(t)\right)-g\left(s(t), \varphi_{i}(t)\right)\right\|^{2} \\
& \quad \leq\left\|x_{j}(t)-s(t)\right\|^{2} h\left(x_{j}(t), s(t)\right) \lambda\left(\varphi_{i}(t)\right) .
\end{aligned}
$$

Here $h(\cdot, \cdot)$ is a known nonnegative continuous function with constant upper bounded $H$, and $\lambda(\cdot)$ is an unknown nonnegative continuous function.

Remark 3. According to separation principle [28], Assumption 2 can be easily satisfied. Under the assumption, we can "separate" the parameters from the nonlinear function. For the unknown parameters, we can solve the problem using adaptive method.

Assumption 4 (Lipschitz condition). For system (1), there exist positive constants $l_{i}>0, i=1, \ldots, N$ such that

$$
\begin{array}{r}
\left\|f_{i}(t, x)-f_{i}(t, y)\right\| \leq l_{i}\|x-y\|, \\
\forall x, y \in R^{n}, \quad i=1,2, \ldots, N .
\end{array}
$$

Assumption 5. In network (1), $\varphi_{i}(t)$ is an unknown timevarying function with a known period $T$; thus, $\lambda\left(\varphi_{i}(t)\right)$ is also a periodic function with the same period $T$. Suppose

$$
\lambda\left(\varphi_{i}(t)\right)=\phi_{i}(t)+\theta_{i},
$$

where $\phi_{i}(t)$ is an unknown continuous function with a known period $T$ and $\theta_{i}$ is an unknown constant parameter.
Assumption 6. In the network (1), the inner coupling matrix $\Gamma$ satisfies

$$
\max \left(\|\Gamma\|, \max \left(\left|\gamma_{i j}\right|\right)\right) \leq \gamma, \quad \forall i, j=1,2, \ldots, N,
$$

where $\gamma$ is positive constant.

Assumption 7. Assume that the state and the state derivative of system (1) are measurable.

Remark 8. This assumption is necessary to design controller and adaptive laws. Assumption 7 seems to be restrictive. The observer for state derivative will be considered in the near future.

Lemma 9 (Young's inequality). For any vectors $x, y \in R^{n}$, and any $c>0$, the following matrix inequality holds:

$$
x^{T} y \leq c x^{T} x+\frac{1}{4 c} y^{T} y .
$$

Lemma 10. For a positive constant $T$, if for all $t \geq 0, f(t)$ is a continuous function, $\int_{t-T}^{t} f(\tau) d \tau$ exists and is bounded with $M$. Then, $f(t)$ is bounded.

Proof. In fact, if $f(t)$ is unbounded, we can get, for all $N>0$, $\exists \tau \in[t-T, t], f(\tau)>N$. As $f(t)$ is a continuous function, according to the properties of the continuous functions, we have $\exists \varepsilon>0$ for all $k \in[\tau-\varepsilon, \tau+\varepsilon] \subset[t-T, t], f(k)>N$; then $\int_{t-T}^{t} f(\tau) d \tau \geq \int_{\tau-\varepsilon}^{\tau+\varepsilon} f(t) d t \geq 2 \varepsilon N$. We can choose $N=$ $M / 2 \varepsilon+1$; then $\int_{t-T}^{t} f(\tau) d \tau>M$. This is a contradiction with the assumption. The proof is completed.

\section{Adaptive Synchronization of the Complex Dynamical Networks}

In this section, adaptive controller is designed to make the states of (1) synchronize to the trajectory $s(t)$, and some sufficient conditions are given to ensure the asymptotic stability of the synchronization process.

For network (1), we design the controller $u_{i}(t) \in R^{n}$ as follows:

$$
\begin{aligned}
u_{i}(t)= & \dot{s}(t)-f_{i}(t, s(t)) \\
& -\gamma H e_{i}(t) \sum_{j=1}^{N} a_{j i}^{2}\left(\widehat{\phi}_{j}(t)+\widehat{\theta}_{j}(t)\right) \\
& -\sum_{j=1}^{N}\left|b_{i j}\right| \gamma \widehat{\alpha}_{i}(t) \operatorname{sign}\left(e_{i}(t)\right)\left|\dot{e}_{j} .(t)\right|,
\end{aligned}
$$

where $\gamma, H$ satisfy Assumption 6, $N$ is the node number of network (1), and $\widehat{\phi}_{i}(t), \widehat{\theta}_{i}(t), \widehat{\alpha}_{i}(t)$ are the estimations of $\phi_{i}(t)$, $\theta_{i}, \alpha_{i}$, respectively. We denote

$$
\begin{gathered}
\operatorname{sign}\left(e_{i}(t)\right)=\left(\operatorname{sign}\left(e_{i 1}(t)\right), \ldots, \operatorname{sign}\left(e_{i n}(t)\right)\right)^{T}, \\
\left|\dot{e}_{j .}(t)\right|=\sum_{k=1}^{n}\left|\dot{e}_{j k}(t)\right| .
\end{gathered}
$$


Remark 11. The main design difficulty is how to deal with the unknown derivative coupling and $\Gamma$. In this paper, we use the known upper bound to magnify the inner coupling matrix. Thus, the controller is related to every element of the synchronization error vector. Under the new controller, we can deal with the derivative terms easily.

The constant parameter distributed update laws are designed as follows:

$$
\begin{gathered}
\dot{\hat{\theta}}_{i}(t)=r_{i} \sum_{j=1}^{N} a_{i j}^{2} e_{j}^{T}(t) e_{j}(t), \\
\dot{\hat{\alpha}}_{i}(t)=g_{i} \sum_{k=1}^{n}\left|e_{i k}(t)\right| \sum_{j=1}^{N}\left|b_{i j}\right|\left|\dot{e}_{j} .(t)\right| .
\end{gathered}
$$

The time-varying distributed periodic adaptive learning law is designed as

$$
\widehat{\phi}_{i}(t)= \begin{cases}0, & t \in[-T, 0), \\ q_{i 0}(t) \sum_{j=1}^{N} a_{i j}^{2} e_{j}^{T}(t) e_{j}(t), & t \in[0, T), \\ \widehat{\phi}_{i}(t-T)+q_{i} \sum_{j=1}^{N} a_{i j}^{2} e_{j}^{T}(t) e_{j}(t), & t \in[T,+\infty),\end{cases}
$$

where $r_{i}, g_{i}, q_{i}$ are positive constants and $q_{i 0}(t)$ is a continuous and strictly increasing nonnegative function which satisfies $q_{i 0}(0)=0, q_{i 0}(T)=q_{i}$, which ensure $\widehat{\phi}_{i}(t)$ is continuous when $t=i T, i=1,2, \ldots, N$.

The following theorem will give a sufficient condition of asymptotical synchronization for the controlled network (3).

Theorem 12. Under Assumptions 2-6, the control law (10) with the distributed adaptive learning laws (12)-(14) guarantees that the controlled network (4) achieves asymptotic synchronization in the $L_{T}^{2}$ norm sense; that is to say,

$$
\lim _{t \rightarrow \infty} \int_{t-T}^{t}\left\|e_{i}(\tau)\right\|^{2} d \tau=0, \quad i=1,2, \ldots, N .
$$

Moreover, all closed-loop signals are bounded.

Proof. Define a Lyapunov-Krasovskii function as follows: for $t \in[0, \infty)$

$$
\begin{aligned}
V(t)= & \frac{1}{2} \sum_{i=1}^{N} e_{i}^{T} e_{i}+\frac{\gamma H}{2} \sum_{i=1}^{N} \int_{t-T}^{t} q_{i}^{-1} \widetilde{\phi}_{i}^{2}(\tau) d \tau \\
& +\frac{\gamma H}{2} \sum_{i=1}^{N} r_{i}^{-1}\left(\widetilde{\theta}_{i}(t)+L\right)^{2}+\frac{\gamma}{2} \sum_{i=1}^{N} g_{i}^{-1} \widetilde{\alpha}_{i}^{2}(t)
\end{aligned}
$$

where

$$
\begin{gathered}
\widetilde{\phi}_{i}(t)=\phi_{i}(t)-\widehat{\phi}_{i}(t), \\
\tilde{\theta}_{i}(t)=\theta_{i}-\widehat{\theta}_{i}(t), \quad \widetilde{\alpha}_{i}(t)=\alpha_{i}-\widehat{\alpha}_{i}(t),
\end{gathered}
$$

and $L$ is a sufficiently large positive constant which will be determined later.

It should be noted that we define

$$
\phi_{i}(t)=\phi_{i}(0), \quad t \in[-T, 0),
$$

from the distributed adaptive law (14), and we get

$$
\widetilde{\phi}_{i}^{2}(t-T)=\phi_{i}^{2}(t-T)=\phi_{i}^{2}(0), \quad t \in[0, T] .
$$

Firstly, the finiteness property of $V(t)$ for the period $[0, T)$ is given. Consider (4) and the proposed distributed control laws (12)-(14), it can be seen that the right-hand side of (4) is continuous with respect to all arguments. According to the existence theorem of differential equation, (4) has unique solution in the interval $\left[0, T_{1}\right) \subset[0, T)$, with $0<T_{1} \leq$ $T$. This can guarantee the boundedness of $V(t)$ over $\left[0, T_{1}\right)$. Therefore, we need only focus on the interval $\left[T_{1}, T\right)$.

For any $\in\left[T_{1}, T\right)$, the derivative of $V(t)$ with respect to time is given by

$$
\begin{aligned}
\dot{V}(t)= & \sum_{i=1}^{N} e_{i}^{T}(t) \dot{e}_{i}(t) \\
& +\frac{\gamma H}{2}\left(\sum_{i=1}^{N} q_{i}^{-1} \widetilde{\phi}_{i}^{2}(t)-\sum_{i=1}^{N} q_{i}^{-1} \widetilde{\phi}_{i}^{2}(t-T)\right) \\
& +\gamma H \sum_{i=1}^{N} r_{i}^{-1}\left(\widetilde{\theta}_{i}(t)+L\right) \dot{\tilde{\theta}}_{i}(t) \\
& +\gamma \sum_{i=1}^{N} g_{i}^{-1} \widetilde{\alpha}_{i}(t) \dot{\tilde{\alpha}}_{i}(t)
\end{aligned}
$$

Let us introduce some notations as

$$
\begin{gathered}
\Phi=f_{i}\left(t, x_{i}(t)\right)-f_{i}(t, s(t)), \\
\Lambda=g\left(x_{j}(t), \varphi_{i}(t)\right)-g\left(s(t), \varphi_{i}(t)\right) .
\end{gathered}
$$

Along the trajectory of (4), we get

$$
\begin{aligned}
& \sum_{i=1}^{N} e_{i}^{T}(t) \dot{e}_{i}(t) \\
&=\sum_{i=1}^{N} e_{i}^{T}(t)\left[f_{i}\left(t, x_{i}(t)\right)+\sum_{j=1}^{N} a_{i j} \Gamma \Lambda\right. \\
&\left.+\sum_{j=1}^{N} \alpha_{i} b_{i j} \Gamma \dot{e}_{j}(t)-\dot{s}(t)+u_{i}(t)\right] .
\end{aligned}
$$


Substituting (10) into the above equation, from Assumption 6 and Lemma 9, we have

$$
\begin{aligned}
& \sum_{i=1}^{N} e_{i}^{T}(t) \dot{e}_{i}(t) \\
& \leq \sum_{i=1}^{N}\left[e_{i}^{T} \Phi+\sum_{j=1}^{N} a_{i j} e_{i}^{T} \Gamma \Lambda+\sum_{j=1}^{N} \alpha_{i} b_{i j} e_{i}^{T} \Gamma \dot{e}_{j}\right. \\
&-\gamma H e_{i}^{T} e_{i} \sum_{j=1}^{N} a_{j i}^{2}\left(\widehat{\phi}_{j}(t)+\widehat{\theta}_{j}(t)\right) \\
&\left.-e_{i}^{T} \sum_{j=1}^{N}\left|b_{i j}\right| \gamma \widehat{\alpha}_{i}(t) \operatorname{sign}\left(e_{i}(t)\right)\left|\dot{e}_{j .}(t)\right| \sum_{j=1}^{N}\right] \\
& \leq \sum_{i=1}^{N}[\left(c e_{i}^{T} e_{i}+\frac{1}{4 c} \Phi^{T} \Phi\right) \\
&+\sum_{j=1}^{N}\left(d e_{i}^{T} e_{i}+\frac{1}{4 d} a_{i j}^{2} \Lambda^{T} \Gamma^{T} \Gamma \Lambda\right) \\
&+\sum_{j=1}^{N} \alpha_{i} b_{i j} e_{i}^{T}(t) \Gamma \dot{e}_{j}(t) \\
&-\gamma H e_{i}^{T} e_{i} \sum_{j=1}^{N} a_{j i}^{2}\left(\widehat{\phi}_{j}(t)+\widehat{\theta}_{j}(t)\right) \\
&\left.-\sum_{j=1}^{N}\left|b_{i j}\right| \gamma \widehat{\alpha}_{i}(t) \sum_{k=1}^{n}\left|e_{i k}(t) \| \dot{e}_{j .}(t)\right|\right] .
\end{aligned}
$$

Using inequality properties, we obtain

$$
\sum_{j=1}^{N} \alpha_{i} b_{i j} e_{i}^{T}(t) \Gamma \dot{e}_{j}(t) \leq \sum_{j=1}^{N}\left|b_{i j}\right| \gamma \alpha_{i} \sum_{k=1}^{n}\left|e_{i k}(t)\right|\left|\dot{e}_{j .}(t)\right| .
$$

Then, according to Assumptions 2-5 and the previous inequality, we have

$$
\begin{aligned}
\sum_{i=1}^{N} e_{i}^{T}(t) \dot{e}_{i}(t) \leq \sum_{i=1}^{N}[ & \left(c e_{i}^{T} e_{i}+\frac{1}{4 c} l_{i}^{2} e_{i}^{T} e_{i}\right) \\
& +\sum_{j=1}^{N} d e_{i}^{T} e_{i}+\sum_{j=1}^{N} a_{i j}^{2}\left(\phi_{i}(t)+\theta_{i}\right) \frac{H \gamma^{2}}{4 d} e_{j}^{T} e_{j} \\
& +\sum_{j=1}^{N}\left|b_{i j}\right| \gamma \alpha_{i} \sum_{k=1}^{n}\left|e_{i k}(t)\right|\left|\dot{e}_{j .}(t)\right| \\
& -\sum_{j=1}^{N} a_{j i}^{2} \gamma H\left(\widehat{\phi}_{j}(t)+\widehat{\theta}_{j}(t)\right) e_{i}^{T} e_{i} \\
& \left.-\sum_{j=1}^{N}\left|b_{i j}\right| \gamma \widehat{\alpha}_{i}(t) \sum_{k=1}^{n}\left|e_{i k}(t)\right|\left|\dot{e}_{j .}(t)\right|\right]
\end{aligned}
$$

where $c$ and $d$ are positive constants. If choosing

$$
c=\frac{1}{2}, \quad d=\frac{\gamma}{4}
$$

we have

$$
\begin{aligned}
\sum_{i=1}^{N} e_{i}^{T}(t) \dot{e}_{i}(t) \leq & \sum_{i=1}^{N}\left(\frac{1}{2}+\frac{1}{2} l_{i}^{2}+\frac{N \gamma}{4}\right) e_{i}^{T} e_{i} \\
& +\sum_{i=1}^{N} \sum_{j=1}^{N} a_{i j}^{2} \gamma H\left(\widetilde{\phi}_{i}(t)+\widetilde{\theta}_{i}(t)\right) e_{j}^{T} e_{j} \\
& +\sum_{j=1}^{N}\left|b_{i j}\right| \gamma \widetilde{\alpha}_{i} \sum_{k=1}^{n}\left|e_{i k}(t)\right|\left|\dot{e}_{j .}(t)\right|
\end{aligned}
$$

From (12), the third term on the right-hand side of (20) satisfies

$$
\gamma H \sum_{i=1}^{N} r_{i}^{-1}\left(\widetilde{\theta}_{i}(t)+L\right) \dot{\tilde{\theta}}_{i}(t)=-\gamma H \sum_{i=1}^{N}\left(\widetilde{\theta}_{i}(t)+L\right) \sum_{j=1}^{N} a_{i j}^{2} e_{j}^{T} e_{j},
$$

while the fourth term on the right-hand side of (20) satisfies

$$
\gamma \sum_{i=1}^{N} g_{i}^{-1} \widetilde{\alpha}_{i}(t) \dot{\tilde{\alpha}}_{i}(t)=-\sum_{i=1}^{N} \gamma \widetilde{\alpha}_{i}(t) \sum_{k=1}^{n}\left|e_{i k}(t)\right| \sum_{j=1}^{N}\left|b_{i j}\right|\left|\dot{e}_{j .}(t)\right| .
$$

Since $q_{i 0}(t)$ is a continuous function and strictly increasing in $\left[T_{1}, T\right) \subset[0, T), q_{i}^{-1} \leq q_{i 0}^{-1}(t)<\infty$ is ensured. Focusing on the second term on the right-hand side of (20), from (19), we can get, when $t \in[0, T)$,

$$
\begin{aligned}
& \frac{\gamma H}{2}\left(\sum_{i=1}^{N} q_{i}^{-1} \widetilde{\phi}_{i}^{2}(t)-\sum_{i=1}^{N} q_{i}^{-1} \widetilde{\phi}_{i}^{2}(t-T)\right) \\
& \leq \frac{\gamma H}{2} \sum_{i=1}^{N} q_{i 0}^{-1} \widetilde{\phi}_{i}^{2}(t) \\
& \quad=\frac{\gamma H}{2} \sum_{i=1}^{N} q_{i 0}^{-1}\left[\phi_{i}^{2}(t)+2 \widehat{\phi}_{i}^{2}(t)-2 \phi_{i}(t) \widehat{\phi}_{i}(t)\right. \\
& \leq \frac{\gamma H}{2} \sum_{i=1}^{N} q_{i 0}^{-1}\left[\phi_{i}^{2}(t)+2 \widehat{\phi}_{i}^{2}(t)-2 \phi_{i}(t) \widehat{\phi}_{i}(t)\right] \\
& =\frac{\gamma H}{2} \sum_{i=1}^{N} q_{i 0}^{-1} \phi_{i}^{2}(t)-\gamma H \sum_{i=1}^{N} \sum_{j=1}^{N} a_{i j}^{2} \widetilde{\phi}_{i}(t) e_{j}^{T} e_{j} .
\end{aligned}
$$

Substituting (27)-(30) into (20), we have

$$
\begin{aligned}
\dot{V}(t) \leq & \sum_{i=1}^{N}\left(\frac{1}{2}+\frac{1}{2} l_{i}^{2}+\frac{N \gamma}{4}-L \sum_{j=1}^{N} a_{j i}^{2}\right) e_{i}^{T} e_{i} \\
& +\frac{\gamma H}{2} \sum_{i=1}^{N} q_{i 0}^{-1}(t) \phi_{i}^{2}(t) .
\end{aligned}
$$


We can choose the appropriate $L$ such that

$$
\sum_{i=1}^{N}\left(\frac{1}{2}+\frac{1}{2} l_{i}^{2}+\frac{N \gamma}{4}-L \sum_{j=1}^{N} a_{j i}^{2}\right)<0 .
$$

According to (31), we have

$$
\dot{V}(t) \leq \frac{\gamma H}{2} \sum_{i=1}^{N} q_{i 0}^{-1}(t) \phi_{i}^{2}(t), \quad t \in[0, T) .
$$

Since $\phi_{i}(t)$ is continuous and periodic, the boundedness can be obtained. The boundedness of $\phi_{i}(t)$ leads to the boundedness of $\dot{V}(t)$. For $V\left(T_{1}\right)$ is bounded, the finiteness of $V(t)$ is obvious, for all $t \in[0, T)$.

Next, we will prove the asymptotical convergence of $e(t)$. According to (16), for all $t \geq T$, we get

$$
\begin{aligned}
\Delta V(t)= & V(t)-V(t-T) \\
= & \frac{1}{2} \sum_{i=1}^{N} e_{i}^{T}(t) e_{i}(t)-\frac{1}{2} \sum_{i=1}^{N} e_{i}^{T}(t-T) e_{i}(t-T) \\
& +\frac{\gamma H}{2} \sum_{i=1}^{N} \int_{t-T}^{t}\left[q_{i}^{-1} \widetilde{\phi}_{i}^{2}(\tau)-q_{i}^{-1} \widetilde{\phi}_{i}^{2}(\tau-T)\right] d \tau \\
& +\frac{\gamma H}{2} \sum_{i=1}^{N} r_{i}^{-1}\left(\widetilde{\theta}_{i}(t)+L\right)^{2} \\
& -\frac{\gamma H}{2} \sum_{i=1}^{N} r_{i}^{-1}\left(\widetilde{\theta}_{i}(t-T)+L\right)^{2}+\frac{\gamma}{2} \sum_{i=1}^{N} g_{i}^{-1} \widetilde{\alpha}_{i}^{2}(t) \\
& -\frac{\gamma}{2} \sum_{i=1}^{N} g_{i}^{-1} \widetilde{\alpha}_{i}^{2}(t-T) .
\end{aligned}
$$

Considering the first two terms on the right-hand side of (34), with Newton-Leibniz formula, we obtain

$$
\begin{aligned}
& \frac{1}{2} \sum_{i=1}^{N} e_{i}^{T}(t) e_{i}(t)-\frac{1}{2} \sum_{i=1}^{N} e_{i}^{T}(t-T) e_{i}(t-T) \\
& =\sum_{i=1}^{N} \int_{t-T}^{t} e_{i}^{T}(\tau) \dot{e}_{i}(\tau) d \tau \\
& \leq \sum_{i=1}^{N} \int_{t-T}^{t}\left(\left(\frac{1}{2}+\frac{1}{2} l_{i}^{2}+\frac{N \gamma}{4}\right) e_{i}^{T}(\tau) e_{i}(\tau)\right. \\
& \quad+\sum_{i=1}^{N} \sum_{j=1}^{N} \int_{t-T}^{t} a_{i j}^{2} \gamma H e_{j}^{T}(\tau) e_{j}(\tau) \tilde{\theta}_{i}^{2}(\tau) d \tau \\
& \left.+\sum_{j=1}^{N} \sum_{j=1}^{N} \int_{t-T}^{t}\left|b_{i j}\right| \gamma e_{j}^{T}(\tau) e_{j}(\tau) \tilde{\phi}_{i}(\tau)\right) d \tau \\
& \quad \sum_{k=1}^{n}\left|e_{i k}(\tau)\right|\left|\dot{e}_{j}(\tau)\right| d \tau
\end{aligned}
$$

Use the algebraic relation

$$
\begin{gathered}
(a-b)^{T} H(a-b)-(a-c)^{T} H(a-c) \\
=(c-b)^{T} H[2(a-b)+(b-c)],
\end{gathered}
$$

where $a, b, c \in R^{p}, H \in R^{p \times p}$.

Choosing $H=1, a=\dot{\phi}_{i}(\tau), b=\widehat{\phi}_{i}(\tau), c=\widehat{\phi}_{i}(\tau-T)$, $\phi_{i}(\tau)=\phi_{i}(\tau-T)$, then $a-c=\widetilde{\phi}_{i}(\tau-T), a-b=\widetilde{\phi}_{i}(\tau)$. The following equality can be obtained:

$$
\begin{aligned}
& \frac{\gamma H}{2} \sum_{i=1}^{N} q_{i}^{-1} \widetilde{\phi}_{i}^{2}(\tau)-\frac{\gamma H}{2} \sum_{i=1}^{N} q_{i}^{-1} \widetilde{\phi}_{i}^{2}(\tau-T) \\
&=\frac{\gamma H}{2} \sum_{i=1}^{N} q_{i}^{-1}\left[2 \widetilde{\phi}_{i}(\tau)+\widehat{\phi}_{i}(\tau)-\widehat{\phi}_{i}(\tau-T)\right] \\
& \times\left[\widehat{\phi}_{i}(\tau-T)-\widehat{\phi}_{i}(\tau)\right] \\
&=-\gamma H \sum_{i=1}^{N}\left(\widetilde{\phi}_{i}(\tau)+\frac{1}{2} q_{i} \sum_{j=1}^{N} a_{i j}^{2} e_{j}^{T} e_{j}\right) \sum_{k=1}^{N} a_{i k}^{2} e_{k}^{T} e_{k} .
\end{aligned}
$$

Taking the third term on the right-hand side of (34), from (37), one obtains

$$
\begin{aligned}
& \frac{\gamma H}{2} \sum_{i=1}^{N} \int_{t-T}^{t}\left[q_{i}^{-1} \tilde{\phi}_{i}^{2}(\tau)-q_{i}^{-1} \tilde{\phi}_{i}^{2}(\tau-T)\right] d \tau \\
& =-\gamma H \sum_{i=1}^{N} \int_{t-T}^{t}\left[\tilde{\phi}_{i}(\tau)+\frac{1}{2} q_{i} \sum_{j=1}^{N} a_{i j}^{2} e_{j}^{T} e_{j}\right] \\
& \times \sum_{k=1}^{N} a_{i k}^{2} e_{k}^{T} e_{k} d \tau .
\end{aligned}
$$

The other terms on right-hand side of (34) can be simplified as follows:

$$
\begin{aligned}
& \frac{\gamma H}{2} \sum_{i=1}^{N} r_{i}^{-1}\left(\widetilde{\theta}_{i}(t)+L\right)^{2} \\
&-\frac{\gamma H}{2} \sum_{i=1}^{N} r_{i}^{-1}\left(\tilde{\theta}_{i}(t-T)+L\right)^{2} \\
&= \gamma H \sum_{i=1}^{N} \int_{t-T}^{t} r_{i}^{-1}\left(\widetilde{\theta}_{i}(\tau)+L\right) \dot{\tilde{\theta}}_{i}(\tau) d \tau \\
&=-\gamma H \sum_{i=1}^{N} \int_{t-T}^{t}\left(\widetilde{\theta}_{i}(\tau)+L\right) \sum_{j=1}^{N} a_{i j}^{2} e_{j}^{T}(\tau) e_{j}(\tau) d \tau, \\
& \frac{\gamma}{2} \sum_{i=1}^{N} g_{i}^{-1} \widetilde{\alpha}_{i}^{2}(t)-\frac{\gamma}{2} \sum_{i=1}^{N} g_{i}^{-1} \widetilde{\alpha}_{i}^{2}(t-T) \\
&=\gamma \sum_{i=1}^{N} \int_{t-T}^{t} g_{i}^{-1} \widetilde{\alpha}_{i}(\tau) \dot{\tilde{\alpha}}_{i}(\tau) d \tau \\
&=-\gamma \sum_{i=1}^{N} \sum_{j=1}^{N} \int_{t-T}^{t}\left|b_{i j}\right| \tilde{\alpha}_{i} \sum_{k=1}^{n}\left|e_{i k}(t)\right|\left|\dot{e}_{j}(t)\right| d \tau .
\end{aligned}
$$




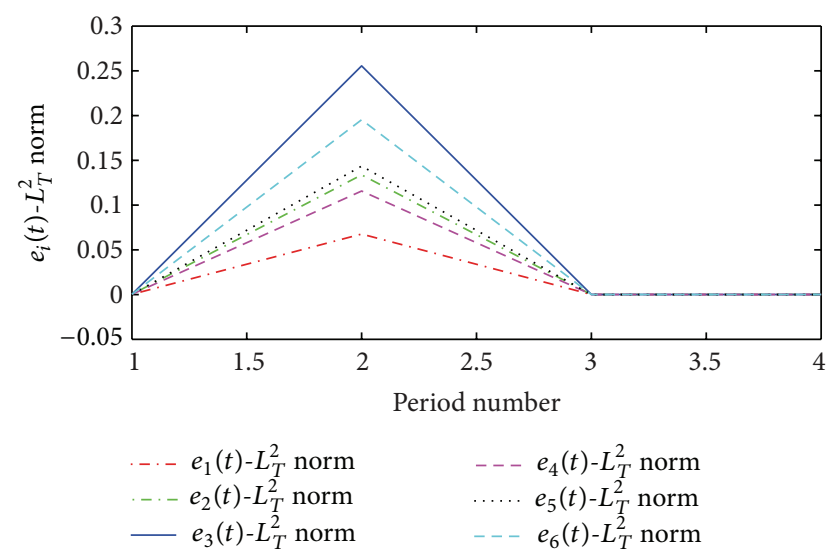

FIgURE 1: The change of synchronization error $e_{i}(t)$ along period number $i=1, \ldots, 6$.

Substituting (35), (38)-(39) into (34), we can attain

$$
\begin{gathered}
\Delta V(t) \leq-\sum_{i=1}^{N} \int_{t-T}^{t}\left(L \sum_{j=1}^{N} a_{j i}^{2}-\frac{1}{2}-\frac{1}{2} l_{i}^{2}-\frac{N \gamma}{4}\right) \\
\times e_{i}^{T}(\tau) e_{i}(\tau) d \tau .
\end{gathered}
$$

Obviously, there exists $L$ such that

$$
L>\frac{\left(1 / 2+(1 / 2) l_{i}^{2}+N \gamma / 4\right)}{\sum_{j=1}^{N} a_{j i}^{2}}, \quad i=1, \ldots, N .
$$

We can obtain

$$
\Delta V(t)<0 .
$$

Applying (40) repeatedly for any $t \in[l T,(l+1) T], l=$ $1,2, \ldots$ and denoting $t_{0}=t-l T$, we have

$$
V(t)=V\left(t_{0}\right)+\sum_{j=0}^{l-1} \Delta V(t-j T) .
$$

Considering $t_{0} \in[0, T)$ and the positive of $V(t)$, according to (43), we obtain

$$
\begin{aligned}
& V(t)<\max _{t_{0} \in[0, T)} V\left(t_{0}\right) \\
& -\sum_{j=0 i=1}^{l-1} \sum_{t-(j+1) T}^{N} \int_{j-j T}^{t-}\left(L \sum_{j=1}^{N} a_{j i}^{2}-\frac{1}{2}-\frac{1}{2} l_{i}^{2}-\frac{N \gamma}{4}\right) \\
& \quad \times e_{i}^{T}(\tau) e_{i}(\tau) d \tau .
\end{aligned}
$$

Since $V\left(t_{0}\right)$ is bounded in the interval $[0, T)$, according to the convergence theorem of the sum of series and (44), the error $e_{i}(t)$ converges to zero asymptotically in the $L_{T}^{2}$ norm sense. That is to say, we have

$$
\lim _{t \rightarrow \infty} \int_{t-T}^{t} e_{i}^{T}(\tau) e_{i}(\tau) d \tau=0
$$

Finally, for all $t \in[T, \infty)$, we prove that all the closed-loop signals are bounded, and the derivative of $V(t)$ is

$$
\begin{aligned}
\dot{V}(t)= & \sum_{i=1}^{N} e_{i}^{T}(t) \dot{e}_{i}(t)+\frac{\gamma H}{2} \sum_{i=1}^{N} q_{i}^{-1} \widetilde{\phi}_{i}^{2}(t)-\frac{\gamma H}{2} \sum_{i=1}^{N} q_{i}^{-1} \widetilde{\phi}_{i}^{2}(t-T) \\
& +\gamma H \sum_{i=1}^{N} r_{i}^{-1}\left(\widetilde{\theta}_{i}(t)+L\right) \dot{\tilde{\theta}}_{i}(t)+\gamma \sum_{i=1}^{N} g_{i}^{-1} \widetilde{\alpha}_{i}(t) \dot{\tilde{\alpha}}_{i}(t) \\
\leq & -\frac{\gamma H}{2} \sum_{i=1}^{N} q_{i}\left(\sum_{j=1}^{N} a_{i j}^{2} e_{j}^{T} e_{j}\right)^{2} \\
& -\sum_{i=1}^{N}\left(L \sum_{j=1}^{N} a_{j i}^{2}-\frac{1}{2}-\frac{1}{2} l_{i}^{2}-\frac{N \gamma}{4}\right) e_{i}^{T} e_{i} .
\end{aligned}
$$

By (46), one can obtain

$$
\begin{aligned}
V(t) \leq & V(T)-\frac{\gamma H}{2} \sum_{i=1}^{N} \int_{T}^{t} q_{i}\left(\sum_{j=1}^{N} a_{i j}^{2} e_{j}^{T} e_{j}\right)^{2} d \tau \\
& -\sum_{i=1}^{N} \int_{T}^{t}\left(L \sum_{j=1}^{N} a_{j i}^{2}-\frac{1}{2}-\frac{1}{2} l_{i}^{2}-\frac{N \gamma}{4}\right) e_{i}^{T} e_{i} d \tau .
\end{aligned}
$$

Choosing

$$
L>\frac{\left(1 / 2+(1 / 2) l_{i}^{2}+N \gamma / 4\right)}{\sum_{j=1}^{N} a_{j i}^{2}}, \quad i=1, \ldots, N
$$

we have

$$
V(t)<V(T) .
$$

From the boundedness of $V(t)$ and (16), we conclude that $e_{i}, \int_{t-T}^{t} \widetilde{\phi}_{i}^{2}(\tau) d \tau, \widetilde{\theta}_{i}(t) \widetilde{\alpha}_{i}(t)$ are all bounded. Since $\widetilde{\phi}_{i}(t)$ is continuous function and $\theta_{i}, \alpha_{i}$ are constants, it implies the boundedness of $\widehat{\theta}_{i}(t)$ and $\widehat{\alpha}_{i}(t)$. According to Lemma 10, the boundedness of $\widetilde{\phi}_{i}(t)$ is obviously obtained. As $\phi_{i}(t)$ is a continuous periodic function, we can get that $\widehat{\phi}_{i}(t)$ is bounded. According to (10), the boundedness of the control input $u_{i}(t)$ is obtained. Since $e_{i}(t)$ is bounded, the boundedness of $x_{i}(t)$ is received. So the proof is completed. 


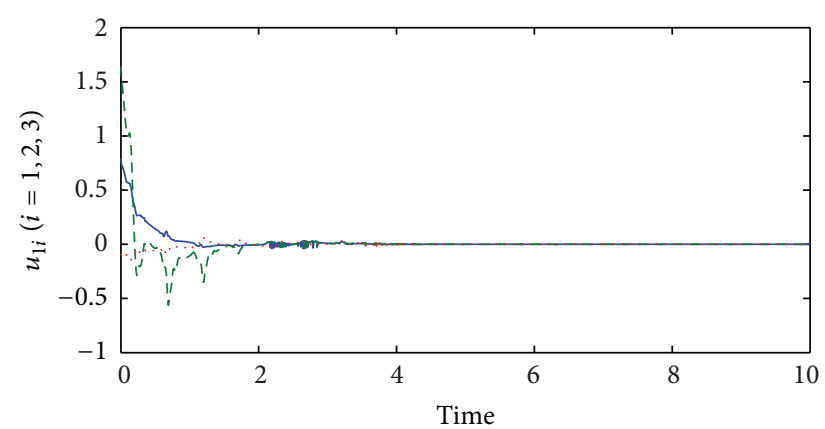

$\begin{array}{ll}- & u_{11} \\ --- & u_{12} \\ \ldots \ldots & u_{13}\end{array}$

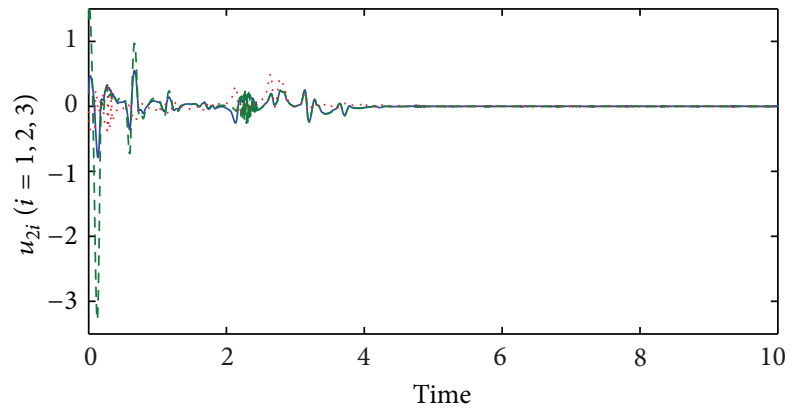

$-u_{21}$

$u_{23}$

(a)

(b)

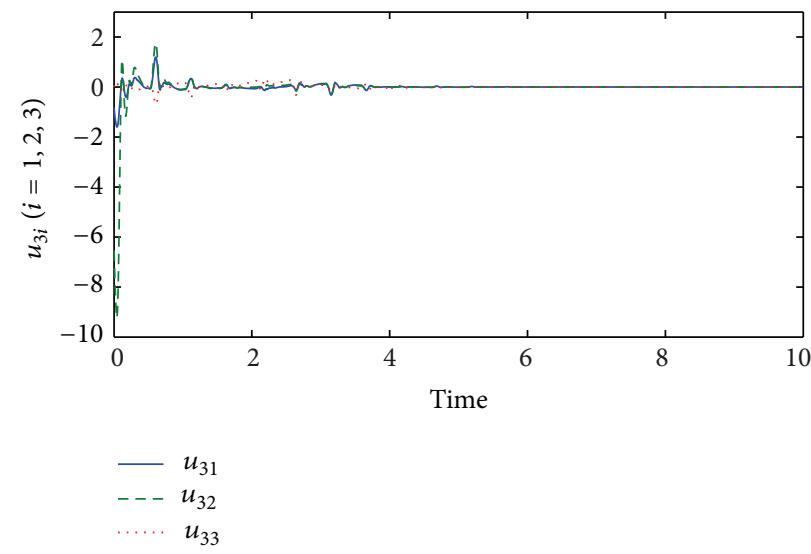

(c)

FIgURE 2: The change of control $u_{i}(t)$ along time: (a) $u_{1 i}, i=1,2,3$, (b) $u_{2 i}, i=1,2,3$, and (c) $u_{3 i}, i=1,2,3$.

\section{Simulation Example}

To demonstrate the theoretical result obtained in Section 3, we consider the following dynamical network with six nonidentical nodes

$$
\begin{aligned}
\dot{x}_{i}(t)= & f_{i}\left(t, x_{i}(t)\right) \\
& +\sum_{j=1}^{N} a_{i j} \Gamma \exp \left(-\varphi_{i}(t)\left(\begin{array}{c}
x_{j 1}^{2}(t) \\
x_{j 2}^{2}(t) \\
x_{j 3}^{2}(t)
\end{array}\right)\right) \\
& +\sum_{j=1}^{N} \alpha_{i} b_{i j} \Gamma \dot{x}_{j}(t)+u_{i}(t), \quad i=1, \ldots, 6,
\end{aligned}
$$

where

$$
f_{1}\left(t, x_{1}\right)=\left(\begin{array}{c}
-2.5 x_{11}+0.3 x_{12}+0.9 x_{13}+3 x_{13}^{2} \\
0.6 x_{11}-2.6 x_{12}+3 x_{12}^{2}+x_{13} \\
-2.8 x_{11}+\sin x_{11} \cos x_{12}-2.2 x_{13}
\end{array}\right)
$$

$$
f_{2}\left(t, x_{2}\right)=\left(\begin{array}{c}
-2.5 x_{21}+x_{22}+x_{21} x_{22}+x_{23} \\
0.5 x_{21}-0.8 x_{22}-\sin x_{22}+x_{23}-x_{23}^{2} \\
-2 x_{21}-0.8 x_{23}-0.5 \sin \left(2 x_{23}\right)
\end{array}\right)
$$$$
f_{3}\left(t, x_{3}\right)=\left(\begin{array}{c}
-2.5 x_{31}+x_{31} \sin x_{31}+x_{32}+1.4 x_{33} \\
0.5 x_{31}-2.5 x_{32}+x_{33} \\
-2 x_{31}+x_{32} x_{33}-0.6 x_{33}-x_{33} \cos x_{33}
\end{array}\right) \text {, }
$$$$
f_{4}\left(t, x_{4}\right)=\left(\begin{array}{c}
-2.5 x_{41}+1.5 x_{42}+1.5 x_{43} \\
x_{41}-2.5 x_{42}+x_{42}^{2}+1.5 x_{43}+x_{43}^{2} \\
-2 x_{41}+x_{41} x_{43}-2.5 x_{43}
\end{array}\right) \text {, }
$$$$
f_{5}\left(t, x_{5}\right)=\left(\begin{array}{c}
-2.1 x_{51}+0.5 x_{51}^{2}+1.4 x_{52}+x_{53} \\
0.9 x_{51}-2.1 x_{52}+x_{52} x_{53}+x_{53} \\
-2 x_{51}+0.4 x_{52}-2.1 x_{53}
\end{array}\right) \text {, }
$$

$$
f_{6}\left(t, x_{6}\right)=
$$

$$
\left(\begin{array}{c}
-3.2 x_{61}+10 x_{62}+2.95\left(\left|x_{61}+1\right|-\left|x_{61}-1\right|\right) \\
x_{61}-x_{62}+x_{63} \\
-14.7 x_{62}
\end{array}\right),
$$



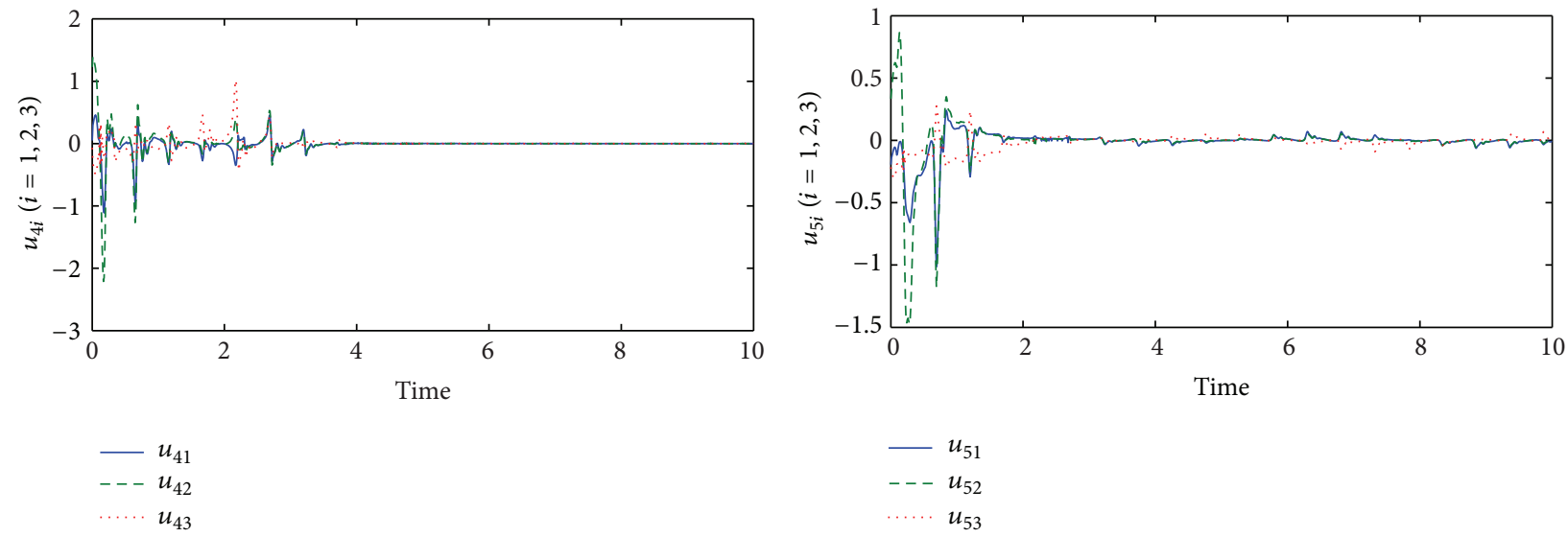

(a)

(b)

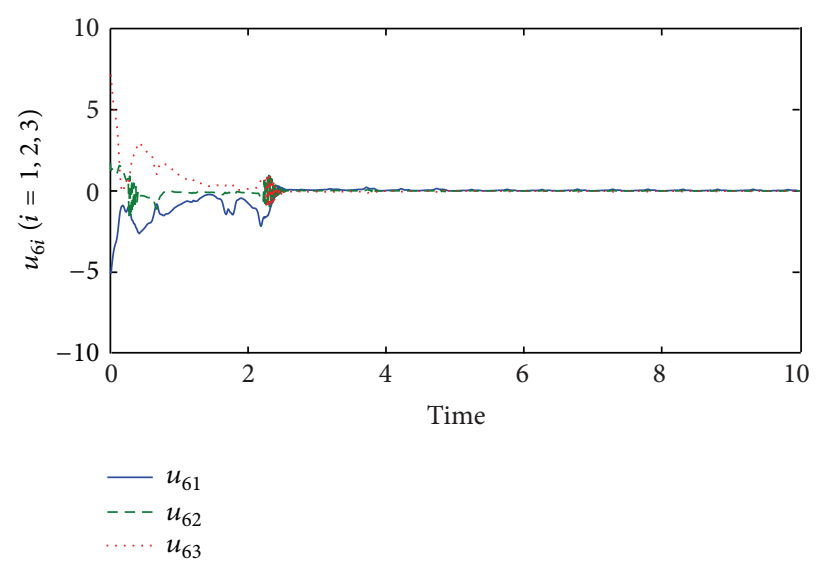

(c)

FIGURE 3: The change of curve of control $u_{i}(t)$ along time: (a) $u_{4 i}, i=1,2,3$, (b) $u_{5 i}, i=1,2,3$, and (c) $u_{6 i}, i=1,2,3$.

$$
\begin{gathered}
\Gamma=\left[\begin{array}{lll}
1 & 0 & 0 \\
0 & 1 & 0 \\
0 & 0 & 1
\end{array}\right], \\
A=\left[\begin{array}{cccccc}
-3 & 0 & 2 & 1 & 0 & 0 \\
2 & -5 & 0 & 0 & 2 & 1 \\
0 & 3 & -4 & 0 & 0 & 1 \\
1 & 1 & 0 & -5 & 2 & 1 \\
0 & 0 & 1 & 3 & -4 & 0 \\
0 & 1 & 1 & 1 & 0 & -3
\end{array}\right], \\
B=\left[\begin{array}{cccccc}
-3 & 2 & 0 & 1 & 0 & 0 \\
0 & -3 & 0 & 0 & 1 & 2 \\
0 & 1 & -3 & 2 & 0 & 0 \\
1 & 0 & 2 & -4 & 0 & 1 \\
0 & 0 & 1 & 1 & -2 & 0 \\
2 & 0 & 0 & 0 & 1 & -3
\end{array}\right] .
\end{gathered}
$$

Nonlinearly parameterized function satisfies

$$
\begin{aligned}
& \left\|\exp \left(-\varphi_{i}(t)\left(\begin{array}{c}
x_{j 1}^{2}(t) \\
x_{j 2}^{2}(t) \\
x_{j 3}^{2}(t)
\end{array}\right)\right)-\exp \left(-\varphi_{i}(t)\left(\begin{array}{c}
s^{2}(t) \\
s^{2}(t) \\
s^{2}(t)
\end{array}\right)\right)\right\|^{2} \\
& \quad \leq\left\|e_{j}(t)\right\|^{2} 2 \varphi_{i}(t) \exp (-1) .
\end{aligned}
$$

We choose $\dot{s}(t)=f_{6}(t, s(t))$, and the parameters are selected as follows:

$$
\begin{array}{ll} 
& N=6, \quad T=0.5, \quad \gamma=1, \quad H=5, \\
& \phi_{1}(t)=0.2 \sin 8 \pi t+2, \quad \phi_{2}(t)=2 \cos 4 \pi t+2, \\
& \phi_{3}(t)=-\sin 8 \pi t+2, \quad \phi_{4}(t)=\cos 8 \pi t+2, \\
& \phi_{5}(t)=-2 \sin 8 \pi t+2, \quad \phi_{6}(t)=2 \sin 4 \pi t+2, \\
& \theta=(1,2,3,1.1,1.5,1.3)^{T}, \\
& \alpha=(0.01,0.01,0.01,0.01,0.01,0.01)^{T} .
\end{array}
$$




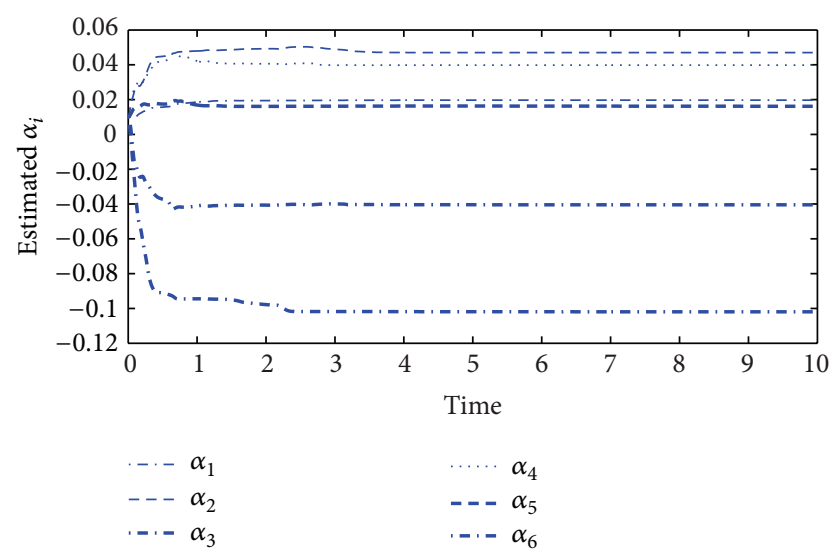

Figure 4: The evaluations of parameters $\alpha_{i}$ along time with $i=$ $1, \ldots, 6$.

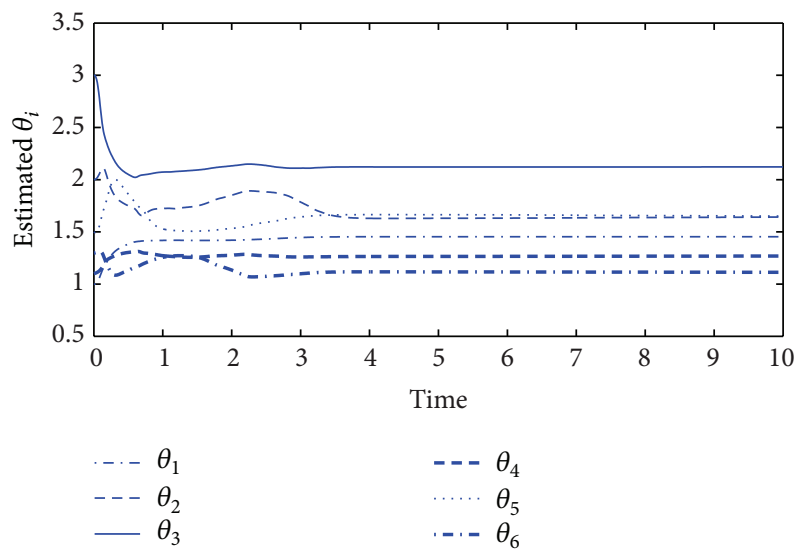

Figure 5: The evaluations of parameters $\theta_{i}$ along time with $i=$ $1, \ldots, 6$.

In the following simulation, we choose

$$
\begin{array}{lll}
q_{1}=1, \quad q_{2}=3, \quad q_{3}=2, & \\
q_{4}=5, \quad q_{5}=1, \quad q_{6}=2, & \\
q_{10}(t)=2 t q_{1}, \quad q_{20}(t)=2 t q_{2}, & q_{30}(t)=2 t q_{3}, \\
q_{40}(t)=2 t q_{4}, \quad q_{50}(t)=2 t q_{5}, & q_{60}(t)=2 t q_{6} .
\end{array}
$$

The initially estimated values of the unknown parameters are

$$
\begin{gathered}
\widehat{\phi}(0)=\left(\begin{array}{llllll}
1 & 1 & 1 & 1 & 1 & 1
\end{array}\right)^{T}, \\
\widehat{\theta}(0)=(1,2,3,1.1,1.5,0)^{T}, \\
\end{gathered}
$$

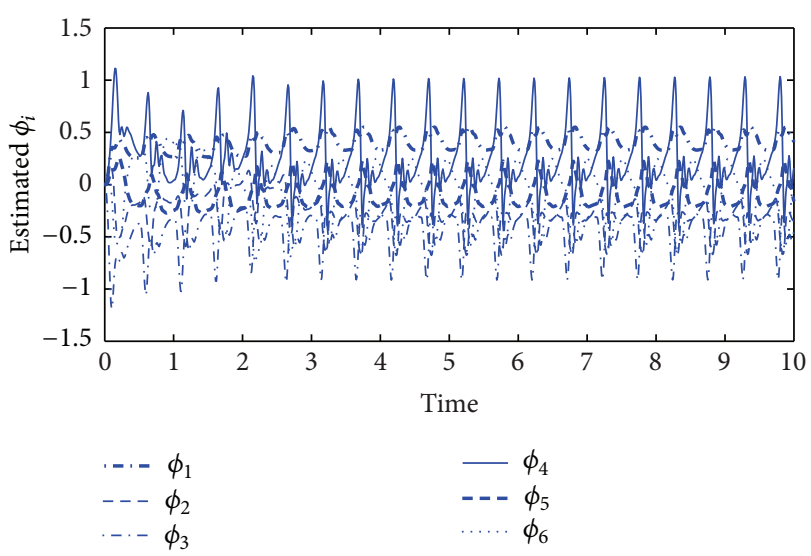

FIgURE 6: The evaluations of parameters $\phi_{i}$ along time with $i=$ $1, \ldots, 6$.

and the initial states are chosen as

$$
\begin{aligned}
& x_{1}=\left[\begin{array}{lll}
0.01 & 0.03 & 0.02
\end{array}\right]^{T}, \quad x_{2}=\left[\begin{array}{lll}
0.03 & 0.02 & 0.03
\end{array}\right]^{T} \text {, } \\
& x_{3}=\left[\begin{array}{lll}
0.04 & 0.02 & 0
\end{array}\right]^{T}, \quad x_{4}=\left[\begin{array}{lll}
0 & 0.05 & 0.03
\end{array}\right]^{T} \text {, }
\end{aligned}
$$

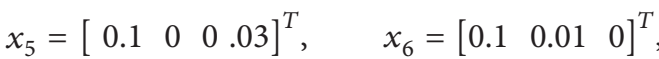

$$
\begin{aligned}
& s=\left[\begin{array}{lll}
0.1 & 0.5 & 0
\end{array}\right]^{T} .
\end{aligned}
$$

According to Theorem 12, the synchronization of the complex dynamical network can be guaranteed by the distributed adaptive controllers (10) and the distributed adaptive learning laws (12)-(14). Figure 1 shows the error evolutions under the designed controllers. Figures 2-3 depict the time evolution of the controllers, and Figures 4, 5, and 6 show the evolution of the estimated time-varying parameters. Figures $2-6$ show that all signals in the network are bounded.

\section{Conclusion}

In this paper, a new distributed adaptive learning control method is applied to the synchronization of complex dynamical networks with nonidentical nodes, nonlinear nonderivative coupling, and derivative coupling. The coupling matrix is not assumed to be symmetric or irreducible. By combining inequality techniques and the parameter separation, introducing the composite energy function, the convergence of the tracking error and the boundedness of the system signals are derived. Simulation results demonstrate the effectiveness of the proposed control method. Future effort is needed to design the observer for state derivative.

\section{Acknowledgment}

This work was supported by the National Natural Science Foundation of China under Grant no. 60974139. 


\section{References}

[1] S. H. Strogatz, "Exploring complex networks," Nature, vol. 410, no. 6825 , pp. 268-276, 2001.

[2] S. Boccaletti, V. Latora, Y. Moreno, M. Chavez, and D.-U. Hwang, "Complex networks: structure and dynamics," Physics Reports, vol. 424, no. 4-5, pp. 175-308, 2006.

[3] J. Lü and G. Chen, "A time-varying complex dynamical network model and its controlled synchronization criteria," IEEE Transactions on Automatic Control, vol. 50, no. 6, pp. 841-846, 2005.

[4] D. H. Ji, S. C. Jeong, J. H. Park, S. M. Lee, and S. C. Won, "Adaptive lag synchronization for uncertain complex dynamical network with delayed coupling," Applied Mathematics and Computation, vol. 218, no. 9, pp. 4872-4880, 2012.

[5] X.-Y. Guo and J.-M. Li, "Stochastic synchronization for timevarying complex dynamical networks," Chinese Physics B, vol. 21, no. 2, Article ID 020501, 2012.

[6] J. Hu, Z. D. Wang, H. J. Gao, and L. K. Stergioulas, "Robust sliding mode control for discrete stochastic systems with mixed time delays, randomly occurring uncertainties, and randomly occurring nonlinearities," IEEE Transactions on Industrial Electronics, vol. 59, no. 7, pp. 3008-3015, 2012.

[7] G.-P. Jiang, W. K.-S. Tang, and G. Chen, "A state-observer-based approach for synchronization in complex dynamical networks," IEEE Transactions on Circuits and Systems I, vol. 53, no. 12, pp. 2739-2745, 2006.

[8] K. Wang, X. Fu, and K. Li, "Cluster synchronization in community networks with nonidentical nodes," Chaos, vol. 19, no. 2, Article ID 023106, 2009.

[9] N. Chopra and M. W. Spong, "Output synchronization of nonlinear systems with time delay in communication," in Proceedings of the 45th IEEE Conference on Decision and Control (CDC '06), pp. 4986-4992, December 2006.

[10] S. Jie, E. M. Bollt, and T. Nishikawa, "Constructing generalized synchronization manifolds by manifold equation," SIAM Journal on Applied Dynamical Systems, vol. 8, no. 1, pp. 202-221, 2009.

[11] J. Xiang and G. Chen, "On the V-stability of complex dynamical networks," Automatica, vol. 43, no. 6, pp. 1049-1057, 2007.

[12] J. G. B. Ramirez and R. Femat, "On the controlled synchronization of dynamical networks with nonidentical nodes," in Proceedings of the 3rd International IEEE Scientific Conference on Physics and Control, pp. 1253-1257, 2007.

[13] J. Zhao, D. J. Hill, and T. Liu, "Synchronization of dynamical networks with nonidentical nodes: criteria and control," IEEE Transactions on Circuits and Systems I, vol. 58, no. 3, pp. 584594, 2011.

[14] T. F. Wang, J. M. Li, and S. Tang, "Adaptive synchronization of nonlinearly parameterized complex dynamical networks with unknown time-varying parameters," Mathematical Problems in Engineering, vol. 2012, Article ID 592539, 16 pages, 2012.

[15] Y. Kuang, Delay Differential Equations with Applications in Population Dynamics, Academic Press, Boston, Mass, USA, 1993.

[16] S.-I. Niculescu, Delay Effects on Stability: A Robust Control Approach, Springer, Berlin, Germany, 2001.

[17] D. W. Gong, H. G. Zhang, Z. S. Wang, and B. Huang, "New global synchronization analysis for complex networks with coupling delay based on a useful inequality," Neural Computing and Applications, vol. 22, no. 2, pp. 205-210, 2013.
[18] Y. H. Xu, W. N. Zhou, J. A. Fang, and W. Sun, "Adaptive synchronization of the complex dynamical network with nonderivative and derivative coupling," Physics Letters A, vol. 374, no. 15-16, pp. 1673-1677, 2010.

[19] J. Wang, L. Feng, and S.-K. Li, "Adaptive synchronization between two delayed complex networks with derivative coupling and non-identical nodes," in Proceedings of the International Conference on Information and Automation (ICIA '11), pp. 135140, June 2011.

[20] Z. Jia, X. C. Fu, G. M. Deng, and K. Z. Li, "Group synchronization in complex dynamical networks with different types of oscillators and adaptive coupling schemes," Communications in Nonlinear Science and Numerical Simulation, vol. 18, no. 10, pp. 2752-2760, 2013.

[21] J. Xiao, Y. H. Yang, and J. S. Long, "Synchronisation of complex networks with derivative coupling via adaptive control," International Journal of Systems Science, vol. 44, no. 12, pp. 2183-2189, 2013.

[22] J. Hu, Z. D. Wang, H. J. Gao, and L. K. Stergioulas, "Robust $\mathrm{H}_{\infty}$ sliding mode control for discrete time-delay systems with stochastic nonlinearities," Journal of the Franklin Institute, vol. 349, no. 4, pp. 1459-1479, 2012.

[23] J. Hu, Z. D. Wang, Y. G. Niu, and L. K. Stergioulas, " $H_{\infty}$ sliding mode observer design for a class of nonlinear discrete timedelay systems: a delay-fractioning approach," International Journal of Robust and Nonlinear Control, vol. 22, no. 16, pp. 18061826, 2012.

[24] J. Hu, Z. D. Wang, and H. J. Gao, "A delay fractioning approach to robust sliding mode control for discrete-time stochastic systems with randomly occurring non-linearities," IMA Journal of Mathematical Control and Information, vol. 28, no. 3, pp. 345363, 2011.

[25] J.-X. Xu and Y. Tan, "A composite energy function-based learning control approach for nonlinear systems with timevarying parametric uncertainties," IEEE Transactions on Automatic Control, vol. 47, no. 11, pp. 1940-1945, 2002.

[26] J. Zhou, J.-A. Lu, and J. H. Lü, "Adaptive synchronization of an uncertain complex dynamical network," IEEE Transactions on Automatic Control, vol. 51, no. 4, pp. 652-656, 2006.

[27] Z. S. Wang and H. G. Zhang, "Synchronization stability in complex interconnected neural networks with nonsymmetric coupling," Neurocomputing, vol. 108, pp. 84-92, 2013.

[28] W. Lin and C. J. Qian, "Adaptive control of nonlinearly parameterized systems: the smooth feedback case," IEEE Transactions on Automatic Control, vol. 47, no. 8, pp. 1249-1266, 2002. 


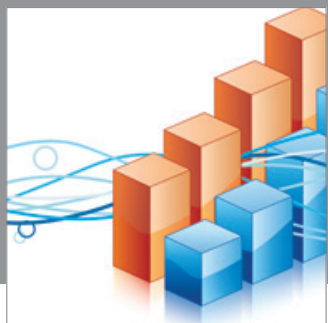

Advances in

Operations Research

mansans

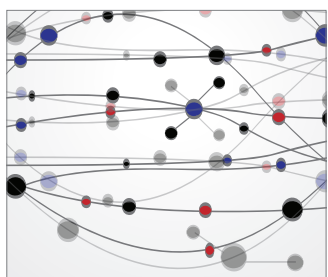

The Scientific World Journal
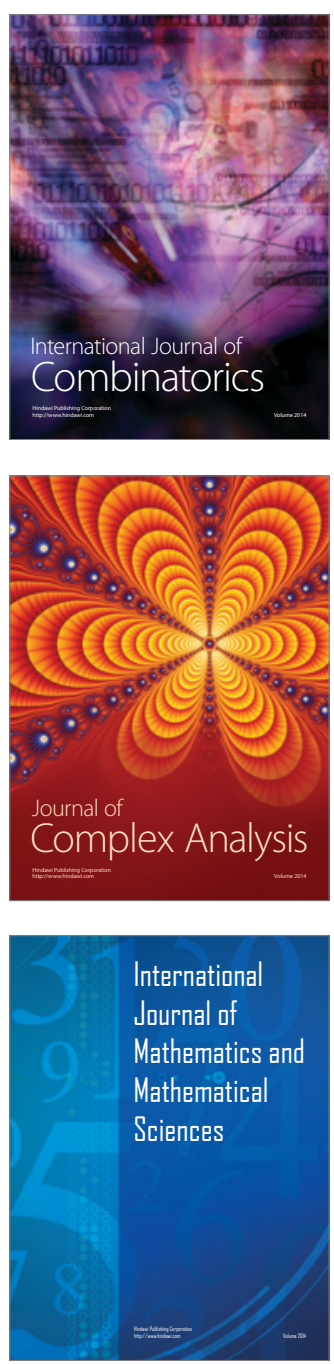
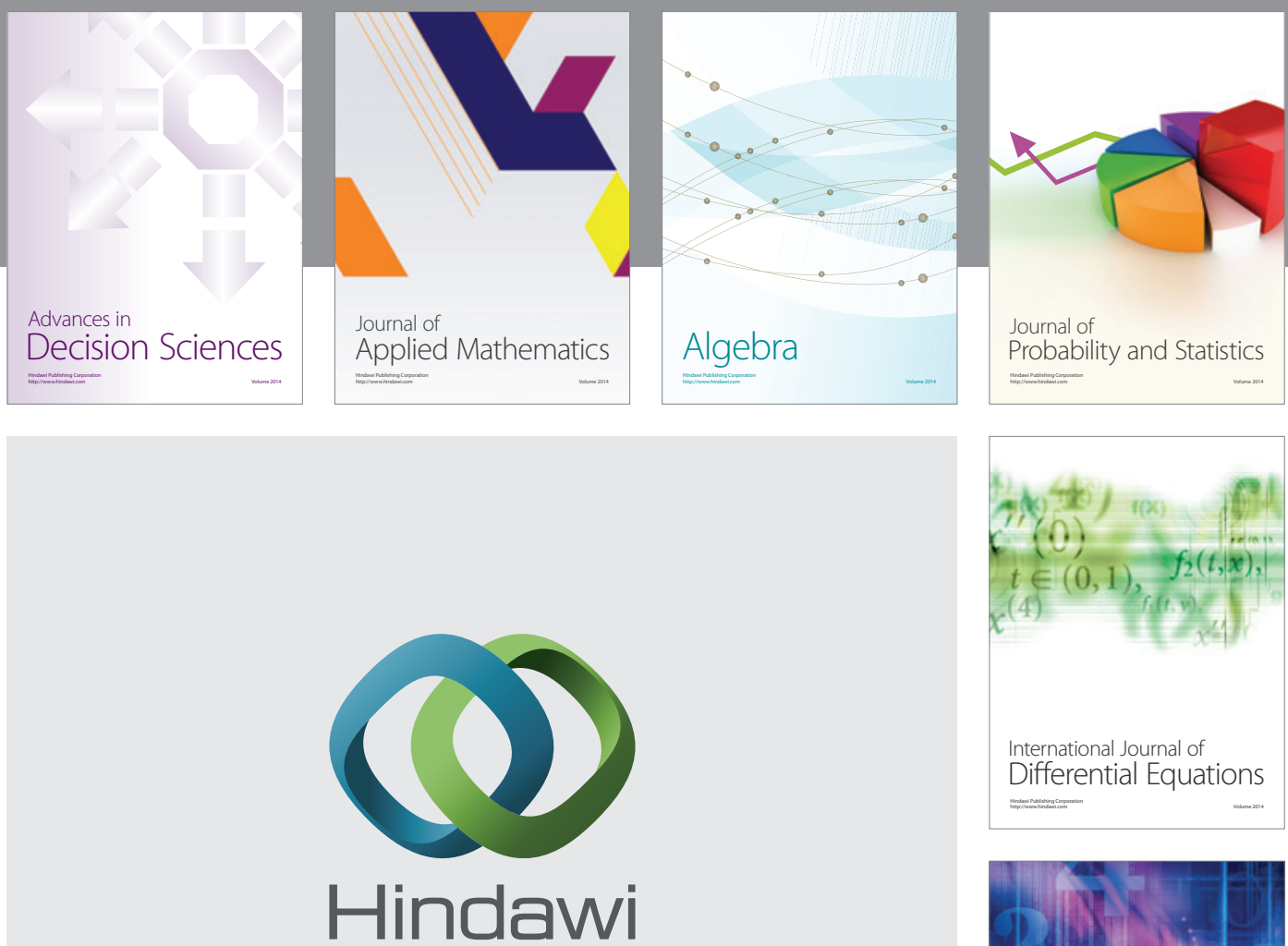

Submit your manuscripts at http://www.hindawi.com
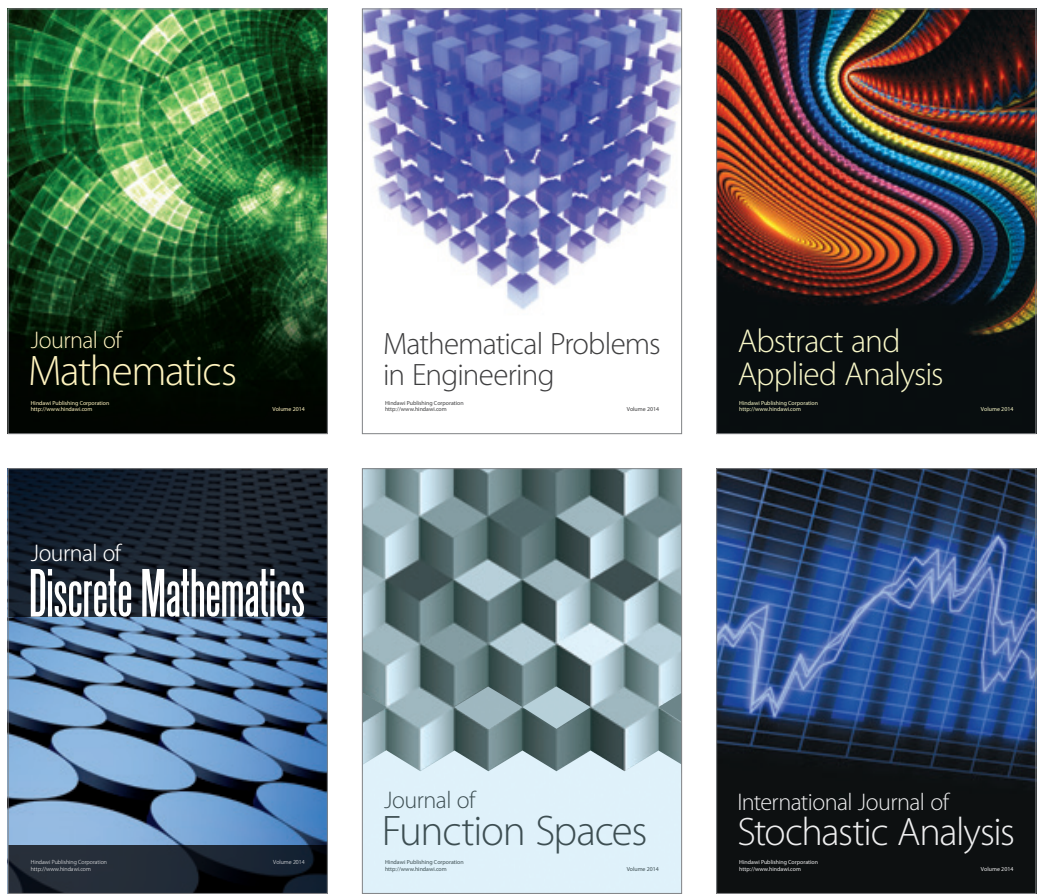

Journal of

Function Spaces

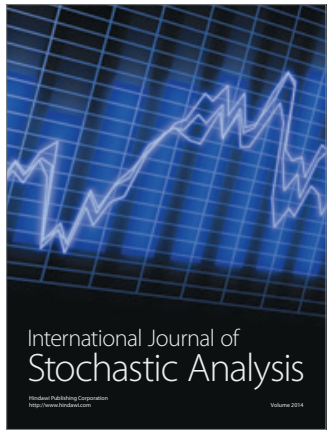

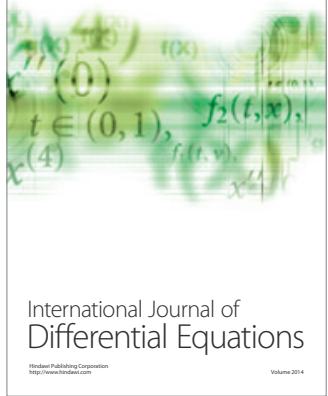
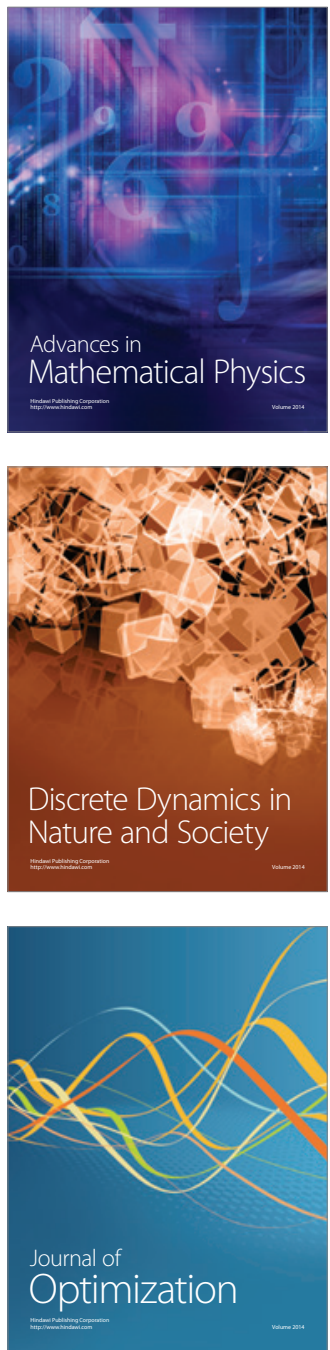